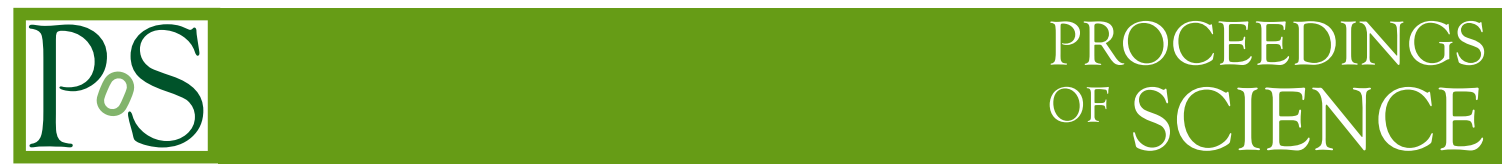

\title{
Challenging the Standard Model at the Tevatron Collider
}

\section{Frank Filthaut ${ }^{* \dagger}$}

Radboud University Nijmegen and Nikhef

E-mail: F.Filthautescience.ru.nl

Even at a time where the world's eyes are focused on the Large Hadron Collider at CERN, which has reached the energy frontier in 2010, many important results are still being obtained from data analyses performed at the Tevatron collider at Fermilab. This contribution discusses recent highlights in the areas of $B$ hadron, electroweak, top quark, and Higgs boson physics.

Kruger 2010: Workshop on Discovery Physics at the LHC

December 5 - 10, 2010

Kruger National Park, Mpumalanga, South Africa

${ }^{*}$ Speaker.
${ }^{\dagger}$ for the $\mathrm{CDF}$ and D0 collaborations 


\section{Introduction}

The standard model (SM) of particle physics forms the cornerstone of our understanding of elementary particles and their interactions, and many of its aspects have been investigated in great detail. Yet it is generally suspected to be incomplete (e.g. by not allowing for the incorporation of gravity in a field theoretical setting) and un-natural (e.g. the mass of the Higgs boson is not well protected against radiative corrections). In addition, it does not explain the dark matter and dark energy content of the Universe. It is therefore of eminent importance to test the limits of validity of the SM.

In the decade since its upgrade to a centre-of-mass energy $\sqrt{s}=1.96 \mathrm{TeV}$, the Tevatron $p \bar{p}$ collider has delivered an integrated luminosity of about $10 \mathrm{fb}^{-1}$, up to $9 \mathrm{fb}^{-1}$ of which are available for analysis by its CDF and D0 collaborations. These large datasets allow for stringent tests of the SM in two areas: direct searches for particles or final states that are not very heavy but that suffer from small production cross sections (e.g. the Higgs boson), and searches for indirect manifestations of beyond-the-standard-model (BSM) effects through virtual effects. The latter searches can often be carried out by precise measurements of otherwise known processes.

This contribution describes such tests of the SM carried out by the CDF and D0 collaborations. In particular, recent highlights in the areas of $B$ hadron physics, electroweak physics, top quark physics, and Higgs boson physics are discussed. Recent results of tests of QCD and of direct searches for new phenomena are described in another contribution [1].

\section{B Hadron Physics}

The $\mathrm{e}^{+} \mathrm{e}^{-} B$ factory experiments Belle and $\mathrm{BaBar}$, with their vast amounts of data collected in the clean environment offered by the $\Upsilon(4 S)$ resonance, have yielded a wealth of information on properties of $B$ hadrons and their decays. These experiments' studies give rise to a very consistent picture of "flavour physics". However, the $\Upsilon(4 S)$ resonance is too light to decay to $B_{s}$ mesons; hence, apart from limited data taken by Belle at the $\Upsilon(5 S)$ resonance, the physics of these mesons is largely the domain of the Tevatron collider. The high integrated luminosity allows for precise studies of exclusive decay modes and provides access to rare $B_{s}$ decays.

\subsection{CP violation in $B \leftrightarrow \bar{B}$ mixing}

Arguably the most noteworthy recent result obtained by the Tevatron experiments in the area of $B$ physics, however, originates from an analysis by the D0 experiment of the inclusive same sign dimuon asymmetry [2]. In final states where both $B$ hadrons decay semileptonically, $B \rightarrow \mu v X$, this measurement is sensitive to $\mathrm{CP}$ violation in $B \leftrightarrow \bar{B}$ mixing; it is therefore interpreted as a measurement of $A_{\mathrm{sl}}^{b} \equiv\left(N_{b}^{++}-N_{b}^{--}\right) /\left(N_{b}^{++}+N_{b}^{--}\right)$involving the semileptonic decays of two $B$ hadrons. The SM prediction for this quantity, $A_{\mathrm{sl}}^{b}=-2.3_{-0.6}^{+0.5} \cdot 10^{-4}$, is too small to be probed by this measurement; therefore any nonzero measurement can be regarded as a sign of new physics.

Besides semileptonic $B$ decays, muons may originate from many other decays. The main background for this analysis is formed by muons resulting from the decay of kaons or pions; or "cascade" $b \rightarrow c \rightarrow \mu X$ decays. For the asymmetry measurement, especially $K^{ \pm}$punch-through (which is more likely to occur for $K^{+}$than for $K^{-}$, due to its higher interaction length) and potential 
muon detection asymmetries could introduce measurement biases. Muon detection asymmetries are tested by regular reversals of the magnet polarities; the fraction of charged kaons misidentified as muons is measured directly in data, using $K^{* 0} \rightarrow K^{+} \pi^{-}$decays. Also other contributions to the asymmetry are estimated directly from data.

The resulting asymmetry is determined to be $A_{\mathrm{sl}}^{b}=-0.00957 \pm 0.00251$ (stat.) \pm 0.00146 (syst.), providing a $3.2 \sigma$ evidence for anomalous $\mathrm{CP}$ violation in $B \leftrightarrow \bar{B}$ mixing. This measurement can be used to constrain the parameters describing CP violation in "wrong-sign" $B_{s}$ meson decays, $a_{\mathrm{sl}}^{s} \equiv\left(\Gamma\left(\bar{B}_{s} \rightarrow \mu^{+} X\right)-\Gamma\left(B_{s} \rightarrow \mu^{-} X\right)\right) /\left(\Gamma\left(\bar{B}_{s} \rightarrow \mu^{+} X\right)+\Gamma\left(B_{s} \rightarrow \mu^{-} X\right)\right)$ and in wrong-sign $B_{d}$ decays, defined analogously. This is shown in Fig. 1.

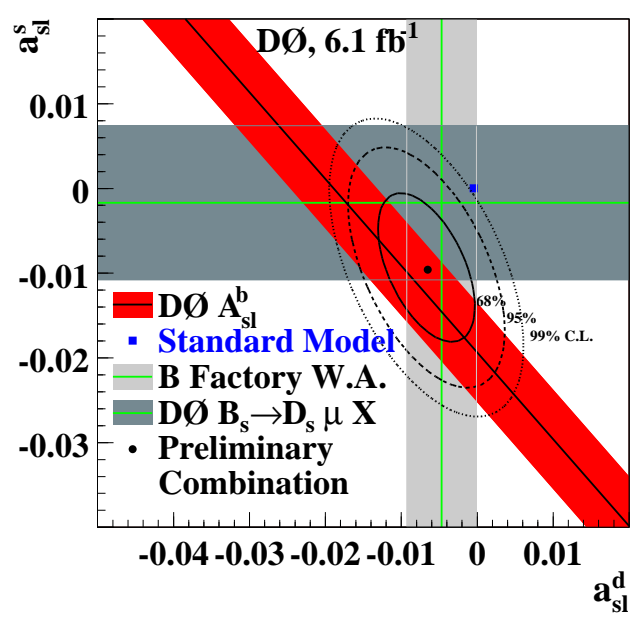

Figure 1: Constraints imposed by D0's measurement of CP violation in same-sign charged muons on the parameters describing $\mathrm{CP}$ violation in wrong-sign $B_{s}$ and $B_{d}$ decays.

\subsection{CP violation in the decay $B_{s} \rightarrow J / \psi \phi$}

From a theoretical point of view, decays of neutral $B$ mesons to $C P$ eigenstates are particularly clean, as CP violation may occur due to the interference between decays of mixed and unmixed $B$ mesons to the same final state. In the case of $b \rightarrow c \bar{c} s$ transitions, as with the decay $B_{s} \rightarrow$ $J / \psi \phi$, such decays can be used to probe the phase $\phi_{s}^{J / \psi \phi} \approx-2 \beta_{s}=2 \arg \left(-V_{t b} V_{t s}^{*} / V_{c b} V_{c s}^{*}\right)$, the SM prediction for which is small, $\approx-0.04$, so that a sizeable observed phase can be interpreted as a sign of BSM contributions.

Both CDF and D0 have used a substantial fraction of their data $\left(5.2\right.$ and $6.1 \mathrm{fb}^{-1}$, respectively) for a measurement of this process, with $\phi \rightarrow K^{+} K^{-}$and $J \psi \rightarrow \mu^{+} \mu^{-}$. Since both the $\phi$ and the $J / \psi$ have $J=1$, final states with different CP need to be separated; this is done using the three-dimensional decay angular distributions in the so-called transversity basis [3]. For this measurement the flavour of the $B_{s}$ meson at the time of production (i.e., $B_{s}$ or $\bar{B}_{s}$ ) needs to be known. Flavour tagging is done by both experiments using opposite-side tagging; CDF also uses same-side kaon tagging. The phase $\phi_{s}^{J / \psi \phi}$ is extracted simultaneously with the difference between the decay widths of the heavy and light $B_{s}$ mass eigenstates, $\Delta \Gamma$. The result of the CDF measurement is 
shown in Fig. 2. The inconsistency with SM predictions that was observed when using only half the data is now largely gone.

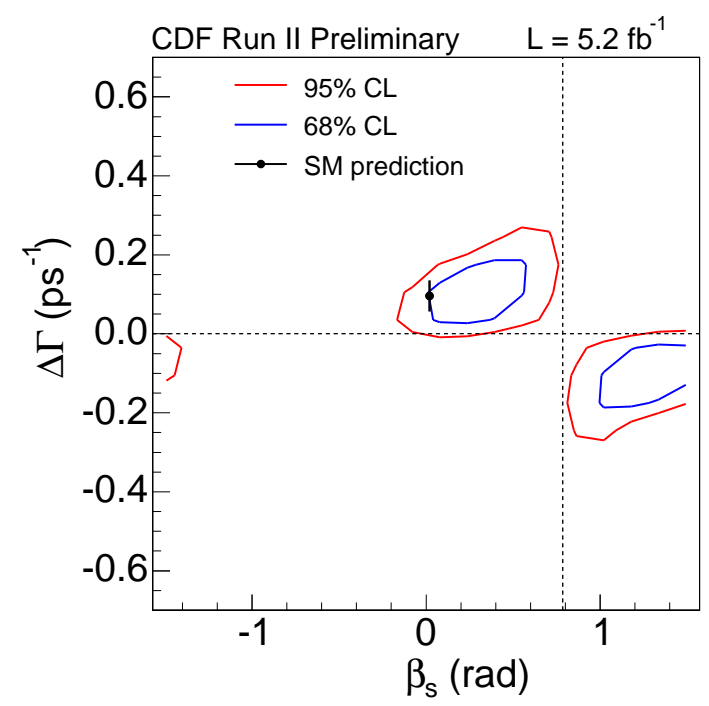

Figure 2: Phase $\phi_{s}^{J \psi \phi}$ and width difference $\Delta \Gamma$ extracted from the CDF $B_{s} \rightarrow J / \psi \phi$ analysis.

\subsection{Rare decays: $B_{d} \rightarrow K^{* 0} \mu^{+} \mu^{-}$}

In the SM, the flavour changing neutral-current (FCNC) process $b \rightarrow s \ell^{+} \ell^{-}$cannot occur through tree level diagrams, and with a branching fraction of order $10^{-6}$ is rare. However, it is also clean theoretically, and has been described in the framework of an Operator Product Expansion, with three dominant Wilson coefficients $C_{7}, C_{9}$, and $C_{10}$ contributing to this process. In the decay $B_{d} \rightarrow K^{* 0} \mu^{+} \mu^{-}$, also the $K^{*}$ polarization is accessible through its decay $K^{* 0} \rightarrow K^{+} \pi^{-}$. Due to its clean experimental signature and the high $B$ production cross section, the statistics available for the study of this decay at the Tevatron are comparable to those at the $\mathrm{e}^{+} \mathrm{e}^{-} B$ factories.

CDF have analyzed [5] this decay using $4.4 \mathrm{fb}^{-1}$ of data, and fit $101 \pm 12$ signal events. The $K^{* 0}$ longitudinal polarization fraction is extracted as a function of dimuon invariant mass $q^{2}$, together with the forward-backward asymmetry of the dimuon system

$$
A_{\mathrm{fb}} \equiv \frac{\sigma\left(\cos \theta_{\mu}>0\right)-\sigma\left(\cos \theta_{\mu}<0\right)}{\sigma\left(\cos \theta_{\mu}>0\right)+\sigma\left(\cos \theta_{\mu}<0\right)}
$$

where $\theta_{\mu}$ is defined as the angle of the $\mu^{+}\left(\mu^{-}\right)$with the opposite of the $B(\bar{B})$ direction in the $\mu^{+} \mu^{-}$rest frame. The results are shown in Fig. 3, and compared to the SM predictions as well as predictions with the $C_{7}$ coefficient changing sign. The results are compatible (and competitive) with those of the $B$ factories, but more statistics is required to probe in detail the SM predictions.

In addition, $\mathrm{CDF}$ have also observed (at a $6.3 \sigma$ significance level) the corresponding decay in the $B_{s}$ system, $B_{s} \rightarrow \phi \mu^{+} \mu^{-}$. The branching fraction for this decay is found to be $(1.44 \pm$ 0.33 (stat.) \pm 0.46 (syst.) $) \cdot 10^{-6}$. 

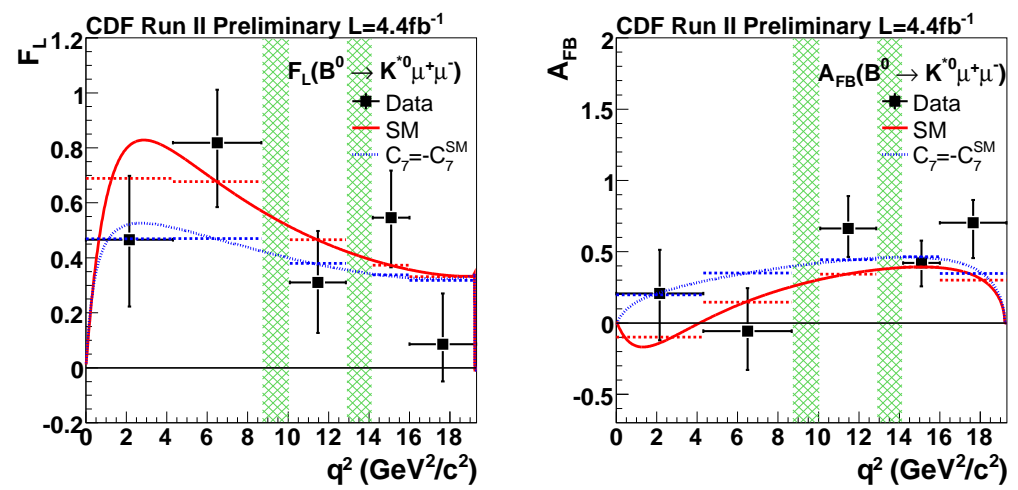

Figure 3: $K^{* 0}$ longitudinal polarization fraction (left) and dimuon forward-backward asymmetry (right) in the decay $B \rightarrow K^{* 0} \mu^{+} \mu^{-}$. The green bands indicate the $J / \psi$ and $\psi^{\prime}$ mass regions, which were excluded from this analysis.

\subsection{Rare decays: $B_{s} \rightarrow \mu^{+} \mu^{-}$}

This decay, too, involves a FCNC process. In the SM it occurs through vertex correction and box diagrams, and its predicted branching fraction is $(3.6 \pm 0.3) \cdot 10^{-9}$. BSM contributions may increase the branching fraction for this process to values well above the SM prediction; e.g. in SUSY models the branching fraction may be enhanced by a factor $\tan ^{6} \beta$, almost independent of SUSY particle masses, and therefore results in sensitivity to the moderate to high $\tan \beta$ regime.

CDF and D0 have searched [6] for this decay in large datasets $\left(3.7 \mathrm{fb}^{-1}\right.$ and $6.1 \mathrm{fb}^{-1}$, respectively). The challenge of this decay mode is to suppress the backgrounds. After requiring that the $\mu^{+} \mu^{-}$pair be compatible with a single decay vertex significantly displaced from the interaction point (to suppress prompt muons), both experiments employ a multivariate analysis using tracking related and kinematic variables. In the D0 analysis, for example, the multivariate analysis is trained using background events from the $m\left(\mu^{+} \mu^{-}\right)$sideband region, and its performance evaluated on $B^{+} \rightarrow J / \psi\left(\rightarrow \mu^{+} \mu^{-}\right) K^{+}$events. The multivariate analysis is subsequently applied to the $B_{s}$ mass region; no significant excess is observed, and an upper limit of $5.1 \cdot 10^{-8}$ at $95 \%$ confidence level (CL) is set on the branching fraction. The corresponding CDF upper limit is slightly lower, $4.3 \cdot 10^{-8}$, to a large extent due to the better mass resolution.

\section{Electroweak Physics}

The area of electroweak physics essentially concerns the (precision) study of (processes involving) the electroweak gauge bosons. This involves both the production of single $W$ and $Z$ bosons and the production of boson pairs. The most notable recent results are presented below.

\subsection{Forward-backward asymmetry in high-mass Drell-Yan production}

While BSM phenomena (and in particular, new gauge bosons in SM extensions) could manifest themselves as additional peaks in the $m\left(\ell^{+} \ell^{-}\right)$spectrum in Drell-Yan events, they could also affect the kinematic distributions in these events. A recent CDF analysis [7] has extended the measurement of the forward-backward asymmetry $A_{\mathrm{FB}}$ in the $e^{+} e^{-}$final states to high $m\left(e^{+} e^{-}\right) \lesssim 600$ 
$\mathrm{GeV}$. In this process, $A_{\mathrm{FB}}$ is defined using the angle of the $e^{-}$with the direction of the quark initiating the interaction, which cannot be determined on an event by event basis. However, in the Tevatron collider's $p \bar{p}$ collisions, unlike the LHC, colliding antiquarks originate mainly from the antiproton, and it is straightforward to associate the quark direction with that of the proton beam. The transverse momenta of the initiating $q$ and $\bar{q}$ are accounted for by employing the Collins-Soper frame.

An earlier D0 analysis of this final state, using a $1.1 \mathrm{fb}^{-1}$ dataset, indicated a hint for a deviation from the SM prediction at the highest $m\left(e^{+} e^{-}\right)$values $\left(250 \mathrm{GeV}<m\left(e^{+} e^{-}\right)<500 \mathrm{GeV}\right)$, but with rather large uncertainties. The CDF analysis uses $4.1 \mathrm{fb}^{-1}$, leading to significantly reduced uncertainties; a very good agreement with the SM predictions is observed.

\section{2 $W$ boson charge asymmetry}

The measurement of the $W$ boson charge asymmetry as a function of the rapidity $y$ of the $W$ boson,

$$
A(y) \equiv \frac{\mathrm{d} \sigma\left(W^{+}\right) / \mathrm{d} y-\mathrm{d} \sigma\left(W^{-}\right) / \mathrm{d} y}{\mathrm{~d} \sigma\left(W^{+}\right) / \mathrm{d} y+\mathrm{d} \sigma\left(W^{-}\right) / \mathrm{d} y},
$$

constitutes a sensitive test of parton density functions: $W$ boson production can proceed via the annihilation of a valence $q \bar{q}^{\prime}$ pair, and as the proton's average up-quark momentum is higher than the average down-quark momentum, $W^{+}\left(W^{-}\right)$bosons are preferably produced along the proton (antiproton) direction. Due to the escaping (anti)neutrino in $W \rightarrow \ell v$ decays, $y$ is not directly accessible. A new D0 analysis [8], performed on a $4 \mathrm{fb}^{-1}$ dataset, instead considers directly the charge asymmetry, as a function of $\eta$, of the $\mu^{ \pm}$produced in $W \rightarrow \mu v$ decays. For low muon $p_{\mathrm{T}}$ and high $|\eta|$ the asymmetry is dominated by the $V-A$ couplings of the $W$ boson; it is for high muon $p_{\mathrm{T}}$ that the $W$ boson charge asymetry (Eqn. 3.1) itself dominates.

The analysis is therefore performed in two bins of muon $p_{\mathrm{T}}: 20 \mathrm{GeV}<p_{\mathrm{T}}<35 \mathrm{GeV}$ and $p_{\mathrm{T}}>35 \mathrm{GeV}$. While the combined asymmetry is compatible with the existing MRST04NLO and CTEQ6.6 predictions [9], the results for $p_{\mathrm{T}}>35 \mathrm{GeV}$ show a marked disagreement, as displayed in Fig. 4.

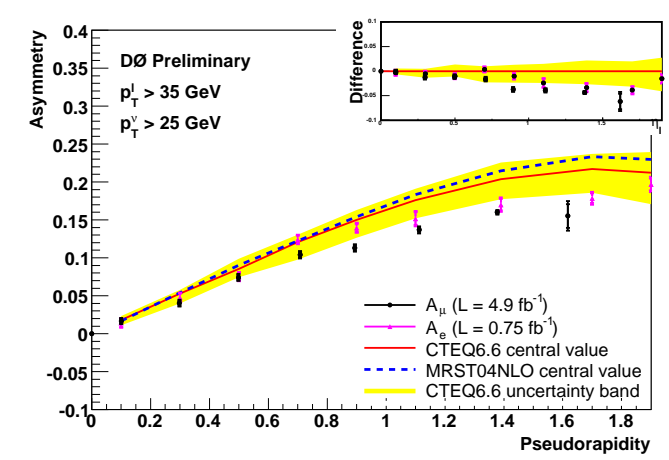

Figure 4: Charge asymmetry measurement in $W \rightarrow \mu v$ decays, for high muon $p_{\mathrm{T}}$. CP symmetry $(A(-\eta)=$ $-A(\eta)$ ) has been verified and subsequently used to represent the measurement as a function of $|\eta|$ only. 


\subsection{Diboson production}

Studying the simultaneous production of two weak gauge bosons $(W, Z)$ is interesting for two reasons: first, many of the resulting final states constitute irreducible backgrounds in searches for the SM Higgs boson; and second, these production processes are sensitive to triple-gauge-boson couplings (TGCs), and hence may be used to probe these TGCs. The general description of these couplings, in terms of contributions from different Lorentz structures, typically requires too many independent parameters to be explored fully. More restrictive assumptions are therefore made. In addition, to preserve unitarity deviations from SM couplings should vanish at high energy, and a cut-off scale $\Lambda$ is usually introduced to ensure this.

The largest production cross section is for $W^{+} W^{-}$production. The cleanest measurements of this production process is in the case of leptonic decays of both $W$ bosons, leading to two charged leptons ( $e$ or $\mu$ ) and two neutrinos. CDF have used a $3.6 \mathrm{fb}^{-1}$ dataset to perform the most precise measurement of the cross section for this production process to date [10], using a matrix element based multivariate analysis. A clean signal results, as shown in Fig. 5, corresponding to a cross section $\sigma\left(p \bar{p} \rightarrow W^{+} W^{-}\right)=12.2 \pm 0.9$ (stat. $)_{-1.4}^{+1.6}$ (syst.) pb, in good agreement with theoretical predictions. D0 have used a $1 \mathrm{fb}^{-1}$ dataset in the same final state to test $W W \gamma$ and $W W Z$ TGCs [11].

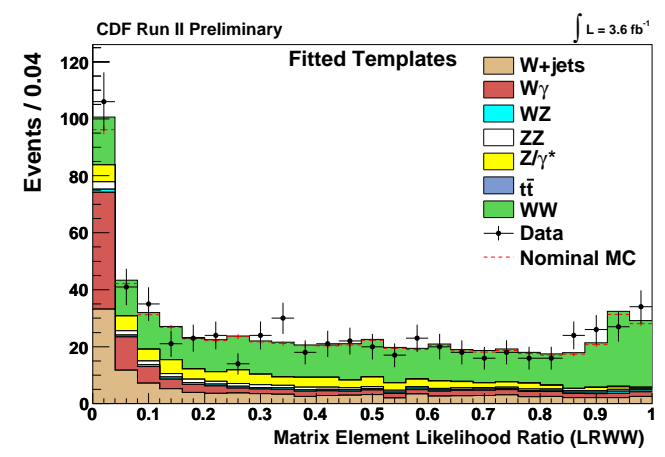

Figure 5: $W^{+} W^{-}$likelihood ratio distribution as measured in CDF data, compared to predictions.

Imposing $S U(2)_{L} \otimes U(1)_{Y}$ symmetry reduces the number of independent coupling constants to three: $\Delta \kappa_{\gamma}, \lambda_{\gamma}$, and $\Delta g_{1}^{Z}$ [12]. No evidence for BSM couplings has been found; their limits, shown in Fig. 6, represent a significant improvement over previous Tevatron analyses.
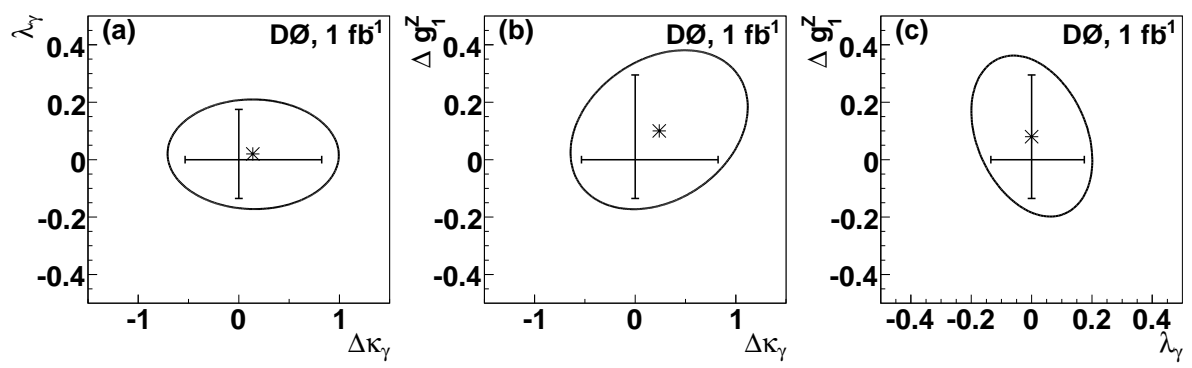

Figure 6: One- and two-dimensional 95\% C.L. limits on BSM TGC constants, assuming a cut-off scale $\Lambda=2 \mathrm{TeV}$, obtained by the D0 analysis [11]. In each case, the constant not viewed is set to zero. The error bars denote the one-dimensional limits. 
The $W Z$ production process suffers from a three times smaller production cross section, but in return is sensitive only to the $W W Z$ TGC. Datasets of $4.1 \mathrm{fb}^{-1}$ and $6 \mathrm{fb}^{-1}$ have been analyzed by D0 [13] and CDF [14], respectively, in the fully leptonic modes (with $\ell=e, \mu$, as above). As shown in Fig. 7, these again lead to clean signals, and have have been used to study the $W W Z$ TGC. In
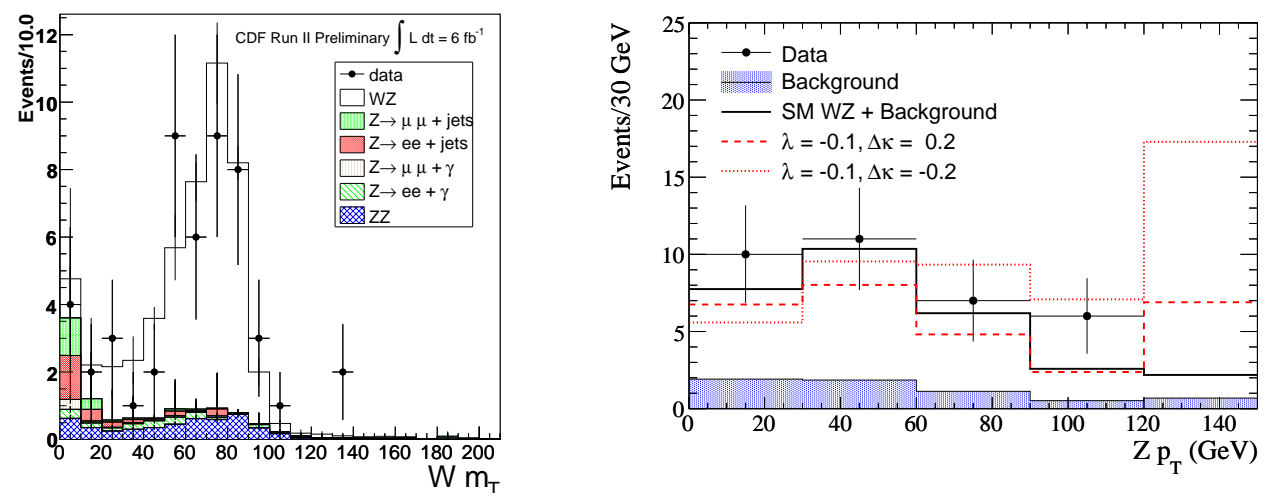

Figure 7: Left: CDF measurement [14] of the $M_{T}$ distribution in $\ell v \ell \ell$ final states, compared to predictions. Right: $Z$ boson $p_{\mathrm{T}}$ distribution compared to SM predictions as well as those in anomalous coupling models [15].

addition, using $2.7 \mathrm{fb}^{-1}$ of data, CDF have also identified $W Z$ and $W W$ production in the $\ell v j j$ final state (the invariant mass resolution is not sufficient to separate cleanly the two processes). With a signal significance of $5.4 \sigma$, this constitutes the first observation of these production processes in this decay mode; the measured combined cross section $\sigma(W W+W Z)=16.0 \pm 3.3 \mathrm{pb}$ is in good agreement with SM predictions.

Finally, also the $Z Z$ production process has been observed in the $Z$ boson leptonic decay modes $\ell^{+} \ell^{-} \ell^{\prime+} \ell^{\prime-}$, by both D0 [17] and CDF [18] using $1.7 \mathrm{fb}^{-1}$ and $1.9 \mathrm{fb}^{-1}$ of data, respectively. In addition, both experiments have also searched for this process in $\ell^{+} \ell^{-} v \bar{v}$ decays [19, 20]. Fig. 8 shows representative results from both final states. In all cases, the observed cross sections are in good agreement with SM predictions. Interpretations in terms of anomalous coupling constants have also been made, but due to the small statistics involved are not yet very restrictive.
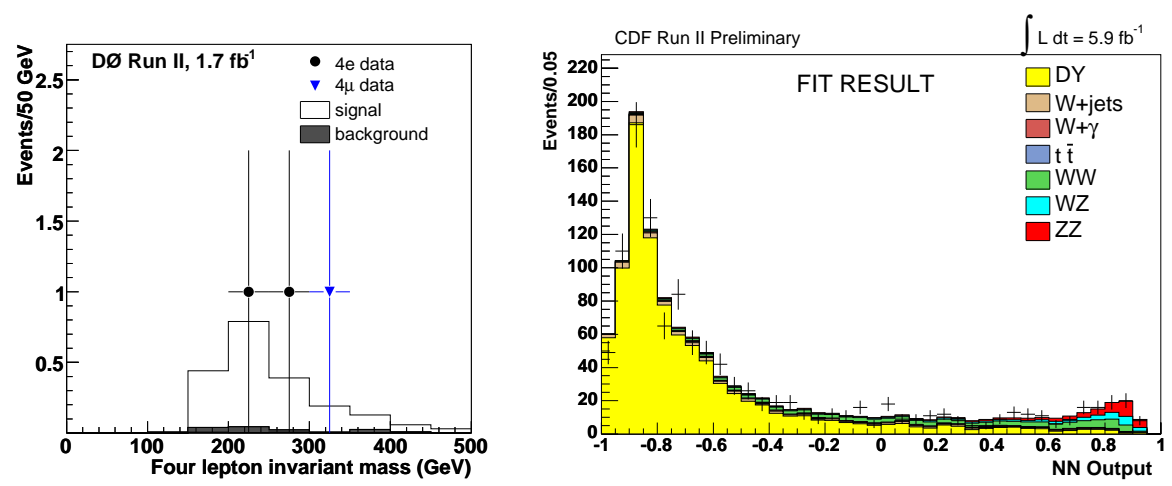

Figure 8: Left: $m\left(\ell^{+} \ell^{-} \ell^{\prime+} \ell^{\prime-}\right)$ distribution in the D0 analysis [17]. Right: discriminant output distribution obtained in the $\operatorname{CDF} \ell^{+} \ell^{-} v \bar{v}$ analysis [20]. 


\section{Top Quark Physics}

Due to its high mass, the top quark manifests itself more distinctly than any other quark, and constitutes an important testing ground for models involving decays to the heaviest particles possible. While suffering from a small cross section (which will lead to the LHC experiments' performance surpassing that of CDF and D0 soon), the high integrated luminosity allows for a number of sensitive studies.

\subsection{Anomalous contributions to $t \bar{t}$ production}

As is the case with e.g. Drell-Yan production (see Sect. 3.1), anomalous contributions to $t \bar{t}$ production could manifest themselves in two ways: first, by modifying the shape of the $m(t \bar{t})$ distribution, and second, by modifying the kinematic distributions of the top quarks. Both of these have been investigated.

Both CDF and D0 have looked for narrow resonances (where the decay width of the resonance does not affect the analysis) in the $m(t \bar{t})$ distribution, in the $\ell+$ jets final state where only one of the $W$ bosons decayed leptonically and the $t \bar{t}$ system can be fully reconstructed. Fig. 9 shows the $m(t \bar{t})$ distribution obtained in the D0 analysis [21] on $3.6 \mathrm{fb}^{-1}$ of data. No evidence for a resonant behaviour is present; exclusion limits depend somewhat on the exact model used but typically, resonances with masses up to $\sim 800 \mathrm{GeV}$ are excluded.
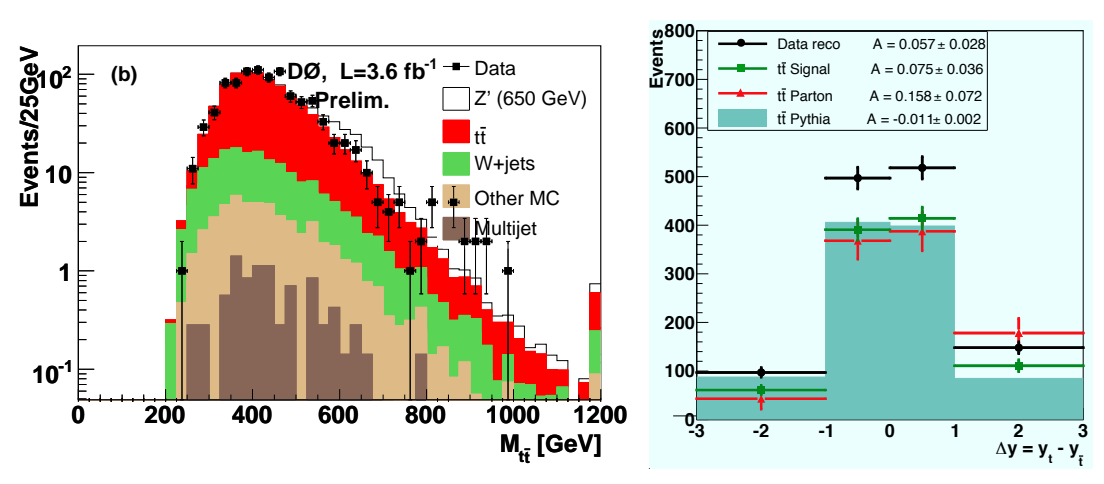

Figure 9: Left: $m(t \bar{t})$ distribution obtained by D0, compared with predictions with and without the presence of a $M=650 \mathrm{GeV} Z^{\prime}$ boson. Right: $A_{\mathrm{FB}}$ measurement by $\mathrm{CDF}$.

Again in $\ell+$ jets events, both experiments have also measured the forward-backward asymmetry $A_{\mathrm{FB}}$, which in the $\mathrm{SM}$ is predicted to be $\sim 0.01$. Here the determination of the $t \bar{t}$ rapidity difference $\Delta y \equiv y_{t}-y_{\bar{t}}$, necessary to distinguish "forward" from "backward" events, suffers from resolution effects and the twofold degeneracy in the solution for the neutrino's $p_{z}$ component. Two approaches have been taken: in the D0 one [22], applied to a $4.3 \mathrm{fb}^{-1}$ dataset, fits are performed directly to observed discriminant distributions for $\Delta y>0$ and $\Delta y<0$ (where a fit is used to determine the event kinematics). The observed asymmetry is somewhat larger than, but still consistent with, the SM prediction. CDF [23], using their $5.3 \mathrm{fb}^{-1}$ dataset, have followed the same approach, but in addition have also unfolded their data to the "true" $\Delta y$ distribution. They find an asymmetry different from the predicted one by more than 2 standard deviations, and that in particular is mostly located at high $|\Delta y|$, as is also shown in Fig. 9. 


\subsection{Fourth generation quarks}

Following the observed pattern in the magnitudes of CKM matrix elements, it is natural to assume that if a fourth generation of (heavy) quarks exists, this will lead primarily to lead to decays to $b$ and $t$ quarks. The precise phenomenology depends on which of the two putative quarks, $b^{\prime}$ or $t^{\prime}$, is lighter.

CDF have searched for such fourth generation quarks, exploiting the signatures of their decays to either $t$ or $b$ through the charged weak interaction and in $\ell+$ jets events. In a search [24] for $t^{\prime} t^{\prime} \rightarrow$ $W^{+} W^{-} b \bar{b}$ decays using a $4.6 \mathrm{fb}^{-1}$ dataset, these decays would manifest themselves predominantly through structures in the reconstructed "top" quark mass distribution and the $H_{\mathrm{T}} \equiv \sum_{j \in \mathrm{jets}} E_{\mathrm{T}, j}+$ $p_{\mathrm{T}, \ell}+\mathbb{E}_{\mathrm{T}}$ distribution. In the absence of a clear signature, $t^{\prime}$ exclusion limits have been set, as shown in Fig. 10. In a search for $b^{\prime} \overline{b^{\prime}} \rightarrow W^{+} W^{-} t \bar{t}$ decays in $4.8 \mathrm{fb}^{-1}$ [25], again higher $H_{\mathrm{T}}$ values are expected than for $t \bar{t}$ final states; however, also higher jet or lepton multiplicities are expected (since in this case both the $b^{\prime}$ and $t$ quark decays lead to $W$ bosons). The search is therefore performed out on the basis of the $H_{\mathrm{T}}$ distributions for five-, six-, and seven-jet events, in different $b$ tag multiplicity bins. The comparison for the double-tagged events is shown in Fig. 10. These results have been used to exclude $b^{\prime}$ quarks with $m_{b^{\prime}}<385 \mathrm{GeV}$.
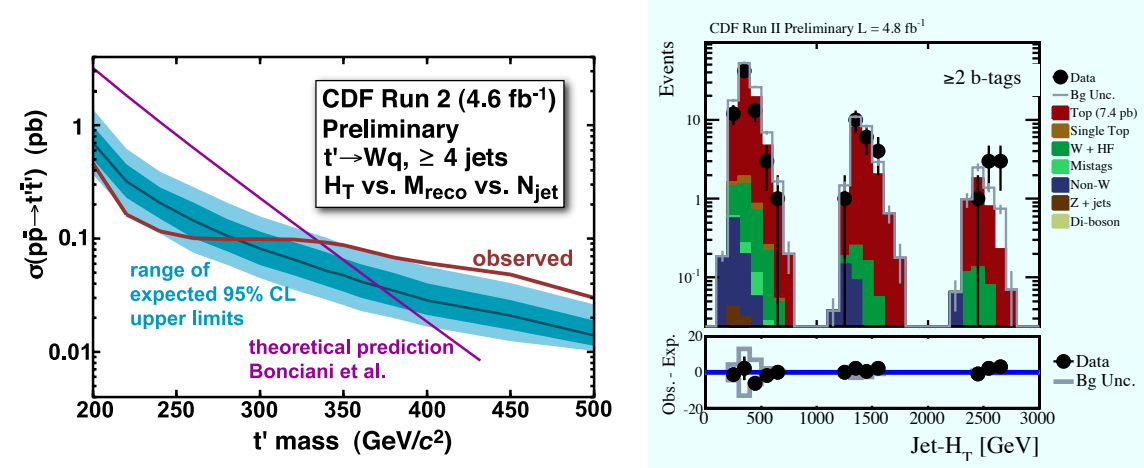

Figure 10: Left: excluded cross section versus $m_{t^{\prime}}$ in the CDF $t^{\prime}$ search. Right: $H_{\mathrm{T}}$ distributions for different jet multiplicities in double-tagged events in the CDF $b^{\prime}$ search. For six-jet (seven-jet) multiplicities the distribution is shifted by $1000 \mathrm{GeV}(2000 \mathrm{GeV})$.

\subsection{Electroweak single top production}

The study of the production of single top quarks in electroweak processes provides an important benchmark for SM Higgs boson searches, to the extent that these processes lead to similar or even identical final states as $W H$ production, but with a larger production cross section. In addition, this production process is interesting in its own right since it allows experimental access to the $\left|V_{t b}\right|$ CKM matrix element without the assumption of three-flavour unitarity.

Using up to $3.2 \mathrm{fb}^{-1}$ of data, both experiments have observed single top quark production [26], and the combined cross section estimates for the so-called $s$ - and $t$-channel processes used to constrain $\left|V_{t b}\right|$ to $\left|V_{t b}\right|=0.91 \pm 0.08$, in good agreement with the constraints from three-flavour unitarity. Both experiments have also analyzed the $s$ - and $t$-channel contributions separately, exploiting the fact that the $t$-channel process often involves a light-quark jet emitted at high $|\eta|$.This has been used in a D0 analysis [27] to probe certain BSM models. 


\section{SM Higgs boson searches and combination}

The search for the SM Higgs boson is the single most important step towards the completion of the SM. Precisely measured variables sensitive to electroweak radiative corrections, and in particular the top quark and $W$ boson masses (measurements of which are presently dominated by the Tevatron experiments), indicate a marked preference for a relatively light Higgs boson, $M_{H}<158$ $\mathrm{GeV}$ at $95 \% \mathrm{CL}$ [28]. The mass range from the LEP limit of $114.4 \mathrm{GeV}$ to this (soft) upper limit matches reasonably well with the optimum experimental sensitivity of the Tevatron direct searches for the Higgs boson.

At moderately high $M_{H} \gtrsim 135 \mathrm{GeV}$, the $H \rightarrow W^{+} W^{-}$decay dominates, and is sufficiently distinctive to be analyzed in the dominant $g g \rightarrow H$ production process. Both experiments have carried out such analyses [29] on datasets of up to $\sim 6 \mathrm{fb}^{-1}$, using the leptonic $W$ boson decay channels. In addition to the clean experimental signature, the analyses benefit from multivariate analysis techniques to improve the separation between the signal and the irreducible non-resonant $W^{+} W^{-}$background; an example from the CDF analysis is shown in Fig. 11. These analyses by themselves have sufficed to exclude (at 95\% CL) a SM Higgs boson with mass $158 \mathrm{GeV}<M_{H}<$ $175 \mathrm{GeV}$, and in the context of a model including a heavy fourth quark family (increasing the $g g \rightarrow H$ production through additional loop contributions) extend the excluded mass region to 131 $\mathrm{GeV}<M_{H}<204 \mathrm{GeV}$.
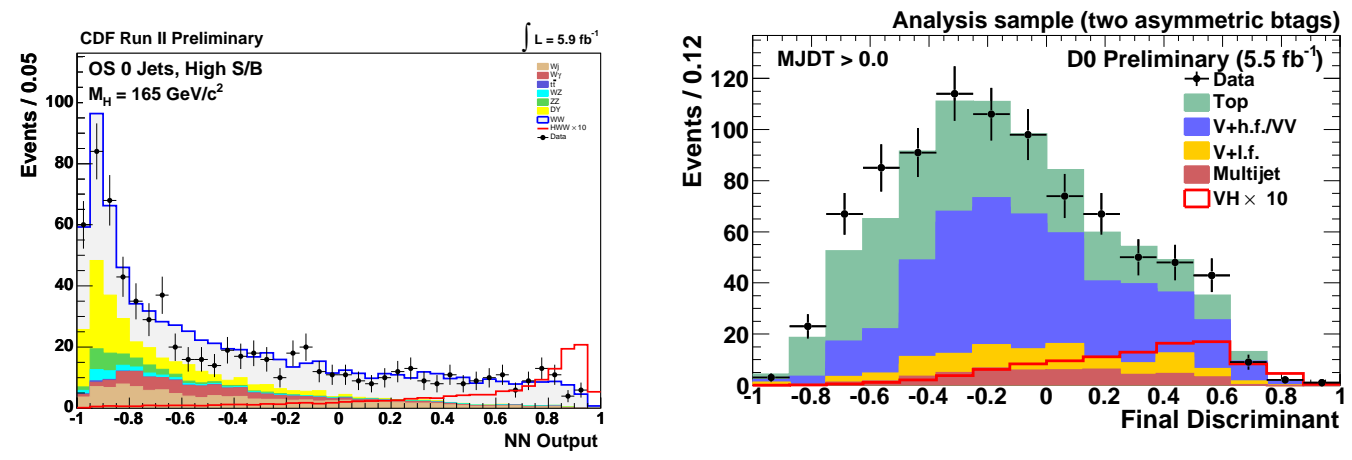

Figure 11: Left: neural network output distribution in the CDF $H \rightarrow W^{+} W^{-}$analysis, compared to background predictions and the expected signal for a $M_{H}=165 \mathrm{GeV}$ SM Higgs boson. Right: neural network output distribution in the D0 $Z H \rightarrow v \bar{v} b \bar{b}$ analysis, compared to background predictions and the expected signal for a $M_{H}=115 \mathrm{GeV}$ SM Higgs boson.

At lower $M_{H}$, the $H \rightarrow b \bar{b}$ decay mode dominates. By itself this signature is not sufficient to identify these decays among the overwhelming QCD $b \bar{b}$ backgrounds; therefore the associated production with a leptonically decaying $W$ or $Z$ boson contributes most to the Higgs boson sensitivity. Also these searches generally employ multivariate techniques, but in addition they must deal with larger instrumental backgrounds, in particular in the $Z H \rightarrow v \bar{v} b \bar{b}$ analysis, as also shown exemplarily in Fig. 11.

The results from all $\sim 100$ search channels are combined. The sensitivity is not yet sufficient to exclude $M_{H}$ values just above the LEP limit, but in addition to the result from the $H \rightarrow W^{+} W^{-}$ searches already confirms the LEP exclusion range $100 \mathrm{GeV}<M_{H}<109 \mathrm{GeV}$. Several analysis 
improvements are upcoming, and further improved sensitivity beyond the mere accumulation of more data is therefore to be expected.

\section{MSSM Higgs bosons}

The Higgs sector is arguably the simplest sector of the minimal supersymmetric standard model (MSSM) describing the phenomenology of supersymetric particles at energies accessible by present colliders. At tree level the MSSM Higgs phenomenology can be described by just two parameters: the ratio of vacuum expectation values of the two Higgs doublets $\tan \beta$, and the mass of any one of the five Higgs bosons $\left(h, H, A, H^{ \pm}\right)$remaining after symmetry breaking; the latter is typically taken to be the mass of the pseudoscalar boson $M_{A}$ for neutral Higgs boson searches, and $M_{H^{ \pm}}$for charged Higgs boson searches. Radiative corrections entail a dependence on other MSSM parameters and are typically incorporated in the form of "scenarios" [30]; they also make it possible for the mass of the lightest Higgs boson, $M_{h}$, to reach values as high as $\sim 135 \mathrm{GeV}$ (this bound is scenario dependent), while at tree level $M_{h}<M_{Z}$.

Tevatron searches for neutral Higgs bosons focus on the high $\tan \beta$ region, where the masses and couplings of the pseudoscalar and one of the neutral scalar Higgs bosons become very similar (these are then generically denoted $\phi$ ), while the remaining neutral scalar Higgs boson tends to decouple. Especially couplings to charged leptons and down-type quarks are enhanced by a factor $\approx \tan \beta$. This has been used to search for $\phi \rightarrow b \bar{b}$ decays [31] produced in association with a third $b$ quark (since, as for the SM Higgs boson, background processes would otherwise obscure this signal; but also because this benefits from the $\sim \tan ^{2} \beta$ dependence of the production cross section). Exclusion limits are set in the $\left(M_{A}, \tan \beta\right)$ plane, as shown for one example in Fig. 12. Both experiments have also searched for the decay $\phi \rightarrow \tau^{+} \tau^{-}$, with at least one of the $\tau$ leptons
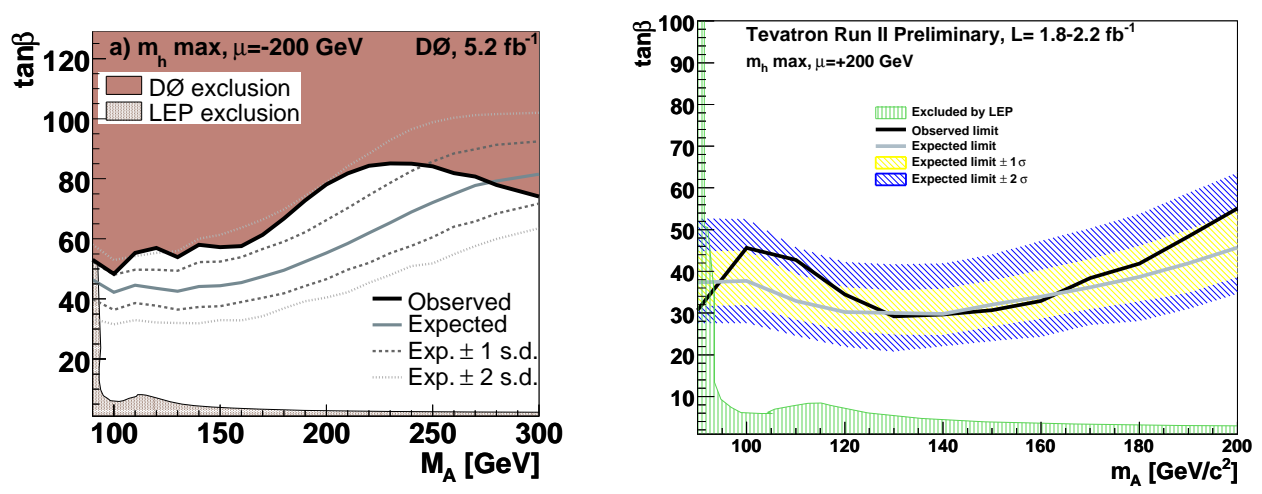

Figure 12: Left: $95 \%$ CL limits on $\left(M_{A}, \tan \beta\right)$ obtained from the D0 search for $\phi \rightarrow b \bar{b}$, in the $m_{h}^{\max }$ scenario. Right: Similar limits from the combined D0 and CDF searches for $\phi \rightarrow \tau^{+} \tau^{-}$decays, again in the $m_{h}^{\max }$ scenario.

decaying leptonically. The resulting final state is sufficiently distinctive to be analyzed in the $g g \rightarrow \phi$ production mode; and this is the most sensitive production channel, even if $b \phi$ production has also been analyzed. The experiments have analyzed datasets up to $2.2 \mathrm{fb}^{-1}$, and set limits in the $\left(M_{A}, \tan \beta\right)$ parameter plane that are tighter (for $M_{A} \lesssim 220 \mathrm{GeV}$ ) and less scenario dependent 
than those for the $\phi \rightarrow b \bar{b}$ searches. The two experiments' $\phi \rightarrow \tau^{+} \tau^{-}$results have also been combined [32] to exclude values of $\tan \beta>30-50$ over a wide $M_{A}$ range, as shown in Fig. 12.

The search for charged Higgs bosons focuses on $M_{H^{ \pm}}$values low enough to allow for $t \rightarrow H^{+} b$ decays. In this case the phenomenology is determined by the $H^{+}$decay, with $H^{+} \rightarrow \tau^{+} v_{\tau}\left(H^{+} \rightarrow\right.$ $c \bar{s})$ dominating for large (small) values of $\tan \beta$. Two types of analysis have been carried out. The first considers the effect of $t \rightarrow H^{+} b$ decays on the branching fractions for $\ell+$ jets, dilepton, and $\ell+\tau$ final states. This has been done in analyses assuming the individual $H^{ \pm}$decay modes; but more powerful is the simultaneous analysis of these final states by D0 [33] using $1 \mathrm{fb}^{-1}$ of data, resulting in $t \rightarrow H^{+} b$ branching fraction limits almost independent of the $H^{ \pm}$decay mode. The resulting excluded region in the $\left(M_{H^{ \pm}}, \tan \beta\right)$ plane is shown in Fig. 13. The second approach
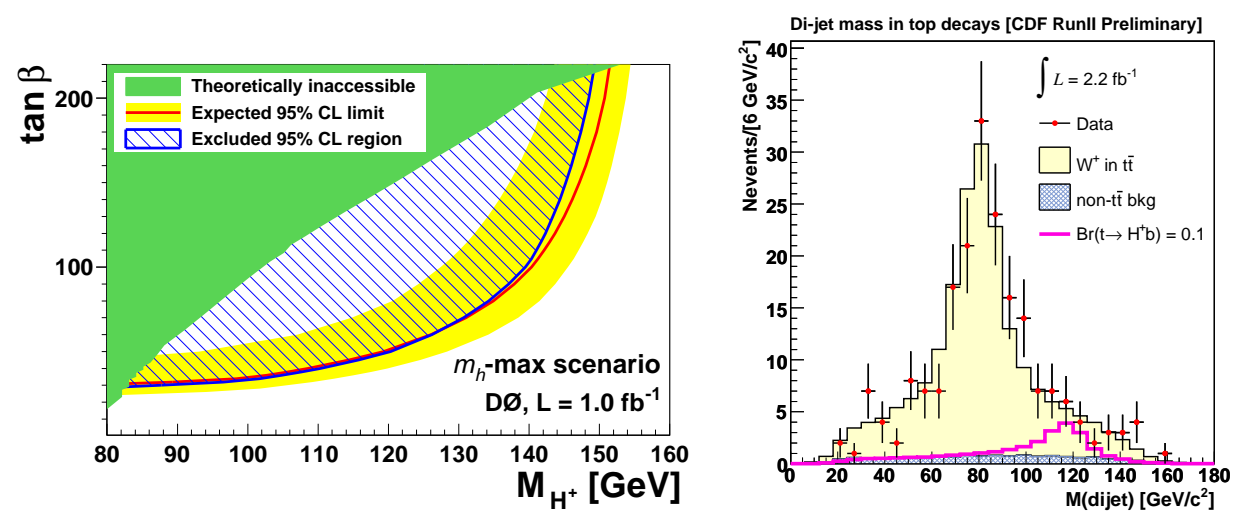

Figure 13: Left: $95 \%$ CL limits on $\left(M_{H^{ \pm}}, \tan \beta\right)$ obtained from the D0 search for $t \rightarrow H^{+} b$ decays in a combination of $t \bar{t}$ decay topologies, in the $m_{h}^{\max }$ scenario. Right: invariant mass spectrum in non- $b$-tagged jets in $\ell+$ jets events in the CDF search for $H^{+} \rightarrow c \bar{s}$ decays.

applies only to $H^{+} \rightarrow c \bar{s}$ decays (and therefore to low values of $\tan \beta$ ), and attempts to search for these decays in $\ell+$ jets events containing exactly four jets through using the invariant mass distribution of the two non- $b$-tagged jets. Assuming $B\left(H^{+} \rightarrow c \bar{s}\right)=1$, a CDF analysis using a $2.2 \mathrm{fb}^{-1}$ dataset [34] limits $B\left(t \rightarrow H^{+} b\right)$ to values less than $\sim 0.1-0.2$, over the mass range of 90-150 GeV considered.

\section{Conclusions}

With a delivered luminosity of about $10 \mathrm{fb}^{-1}$, The Tevatron collider has provided a wealth of data useful both for precise studies of "known" final states and for direct searches for new particles. Analyses by the CDF and D0 collaborations have exploited datasets of up to $\sim 6 \mathrm{fb}^{-1}$ to test the SM and search for evidence for BSM phenomena in the areas of $B$ hadron physics, electroweak physics, top quark physics, and Higgs boson physics. Many analyses are still in progress, and more precise results and stringent tests are to be expected in the coming few years, even after Tevatron operation will cease. The Tevatron collider and its experiments have been very successful in their endeavours. I wish the LHC and its experiments equally exciting times! 


\section{References}

[1] J. Deng, Search for New Physics Beyond SM at the Tevatron, these proceedings.

[2] D0 Collaboration, V.M. Abazov et al., Evidence for an Anomalous Like-Sign Dimuon Charge Asymmetry, Phys. Rev. D82 (2010) 032001; Phys. Rev. Lett. 105 (2010) 081801.

[3] A.S. Dighe, I. Dunietz, and R. Fleischer, Extracting CKM phases and $B_{s}-\bar{B}_{s}$ mixing parameters from angular distributions of nonleptonic B decays, Eur. Phys. J. C6 (1999) 647; I. Dunietz, R. Fleischer, and U. Nierste, In pursuit of new physics with $B_{s}$ decays, Phys. Rev. D63 (2001) 114015.

[4] CDF Collaboration, An Updated Measurement of the CP Violating Phase $\beta^{J / \psi \phi}$ in $B^{0} \rightarrow J / \psi \phi$ Decays using 5.2 $\mathrm{fb}^{-1}$ of Integrated Luminosity, CDF Public Note 10206, 2010.

[5] CDF Collaboration, Measurement of Forward-Backward Asymmetry in $B \rightarrow K^{(*)} \mu^{+} \mu^{-}$and First Observation of $B_{s}^{0} \rightarrow \phi \mu^{+} \mu^{-}$, submitted to Phys. Rev. Lett., e-print 1101.1028 [hep-ex].

[6] CDF Collaboration, Search for $B_{s}^{0} \rightarrow \mu^{+} \mu^{-}$and $B_{d}^{0} \rightarrow \mu^{+} \mu^{-}$Decays in $3.7 \mathrm{fb}^{-1}$ of p p $\bar{p}$ Collisions with CDF II, CDF Public Note 9892, 2009; D0 Collaboration, Search for the rare decay $B_{s}^{0} \rightarrow \mu^{+} \mu^{-}$, Phys. Lett. B693 (2010) 539.

[7] CDF Collaboration, Forward-Backward Charge Asymmetry of High Mass Drell-Yan Dielectron Pairs, CDF Public Note, 2009.

[8] D0 Collaboration, Measurement of the muon charge asymmetry in $p \bar{p} \rightarrow W+X \rightarrow \mu \nu+X$ events using the D0 detector, D0 Note 5976-CONF.

[9] P.M. Nadolsky et al., Implications of CTEQ global analysis for collider observables, Phys. Rev. D78 (2008) 013004; A.D. Martin, R.G. Roberts, W.J. Stirling, and R.S. Thorne, Physical Gluons and High-E $E_{T}$ Jets, Phys. Lett. B604 (2004) 61.

[10] CDF Collaboration, Measurement of the WW Production Cross Section in p $\bar{p}$ Collisions at $\sqrt{s}=1.96$ TeV using 3.6 $\mathrm{fb}^{-1}$ of CDF Run II Data, CDF Public Note 9753, 2009.

[11] D0, Collaboration, Measurement of the $W W$ production cross section with dilepton final states in $p \bar{p}$ collisions at $\sqrt{s}=1.96 \mathrm{TeV}$ and limits on anomalous trilinear gauge couplings, Phys. Rev. Lett. 103 (2009) 191801.

[12] K. Hagiwara, R. Peccei, and D. Zeppenfeld, Probing the Weak Boson Sector in $e^{+} e^{-} \rightarrow W^{+} W^{-}$, Nucl. Phys. B282 (1987) 253; K. Hagiwara, D. Woodside, and D. Zeppenfeld, Measuring the WWZ Coupling at the Tevatron, Phys. Rev. D41 (1990) 2113.

[13] D0 Collaboration, Measurement of the $W Z \rightarrow \ell v \ell \ell$ cross section and limits on anomalous triple gauge couplings in p $\bar{p}$ collisions at $\sqrt{s}=1.96 \mathrm{TeV}$, submitted to Phys. Lett. B.

[14] CDF Collaboration, Measurement of the WZ and ZZ Production Cross Sections Using the $W Z \rightarrow 3 \ell+\mathbb{E}_{T}$ and $Z Z \rightarrow 4 \ell$ Channels, CDF Public Note 10238 (2010).

[15] K. Hagiwara, S. Ishihara, R. Szalapski, and D. Zeppenfeld, Low-energy effects of new interactions in the electroweak boson sector, Phys. Rev. D48 (1993) 2182.

[16] CDF Collaboration, Measurement of the $W W+W Z$ Production Cross Section Using the Lepton+Jets Final State at CDF II, Phys. Rev. Lett. 104 (2010) 101801.

[17] D0 Collaboration, Observation of ZZ production in p $\bar{p}$ collisions at $\sqrt{s}=1.96 \mathrm{TeV}$, Phys. Rev. Lett. 101 (2008) 171803.

[18] CDF Collaboration, Observation of $Z Z \rightarrow l l l^{\prime} l^{\prime}$ at $C D F$, CDF Public Note 9910, 2009. 
[19] D0 Collaboration, $Z Z \rightarrow l^{+} l^{-} v \bar{v}$ production in $p \bar{p}$ collisions at $\sqrt{s}=1.96 \mathrm{TeV}$, Phys. Rev. D78 (2008) 072002.

[20] CDF Collaboration, $Z^{0} Z^{0}$ cross section measurement in $l l v \bar{v}$ decay channel at CDF, CDF Public Note 10358, 2010.

[21] D0 Collaboration, Search for $t \bar{t}$ resonances in the lepton+jets final state in p $\bar{p}$ collisions at $\sqrt{s}=1.96$ $\mathrm{TeV}, \mathrm{D} 0$ Note 5882-CONF.

[22] D0 Collaboration, Measurement of the forward-backward production asymmetry of t and $\bar{t}$ quarks in $p \bar{p} \rightarrow t \bar{t}$ events, D0 Note 6062-CONF, 2010.

[23] CDF, Collaboration, Measurement of the Inclusive Forward-Backward Asymmetry and its Rapidity Dependence $A_{\mathrm{fb}}(\Delta y)$ in $\bar{t} t$ Production in $5.3 \mathrm{fb}^{-1}$ of Tevatron Data, CDF Public Note 10224, 2010.

[24] CDF Collaboration, Search for Heavy Top $t^{\prime} \rightarrow W q$ in Lepton Plus Jets Events in $\int \mathscr{L} \mathrm{d} t=4.6 \mathrm{fb}^{-1}$, CDF Public Note 10110, 2010.

[25] CDF Collaboration, Search for heavy bottom-like chiral quarks decaying to an electron or muon and jets, CDF Public Note 10243, 2010.

[26] D0 Collaboration,Observation of Single Top-Quark Production, Phys. Rev. Lett. 103 (2009) 092001; CDF Collaboration, First Observation of Electroweak Single Top Quark Production, Phys. Rev. Lett. 103 (2009) 092002.

[27] D0 Collaboration, Measurement of the t-channel single top quark production cross section, Phys. Lett. B682 (2010) 363.

[28] LEP, SLD, and Tevatron Electroweak Working Groups, Precision Electroweak Measurements and Constraints on the Standard Model, CDF Public Note 10338, D0 Note 6130, 2010.

[29] CDF Collaboration, Search for $H \rightarrow W W^{*}$ Production at CDF Using $5.9 \mathrm{fb}^{-1}$ of Data, CDF Public Note 10232, 2010; D0 Collaboration, Search for Higgs boson production in dilepton and missing energy final states with $5.4 \mathrm{fb}^{-1}$ of p $\bar{p}$ collisions at $\sqrt{\mathrm{s}}=1.96 \mathrm{TeV}$, Phys. Rev. Lett. 104 (2010) 061804; CDF and D0 Collaborations, Combined Tevatron Upper Limit on $g g \rightarrow H \rightarrow W^{+} W$ and Constraints on the Higgs Boson Mass in Fourth-Generation Fermion Models, Phys. Rev. D82 (2010) 011102.

[30] M. Carena, S. Heinemeyer, C.E.M. Wagner, and G. Weiglein, Suggestions for benchmark scenarios for MSSM Higgs boson searches at hadron colliders, Eur. Phys. J. C26 (2003) 601.

[31] D0 Collaboration, Search for neutral Higgs bosons in the multi-b-jet topology in $5.2 \mathrm{fb}^{-1}$ of p $\bar{p}$ collisions at $\sqrt{s}=1.96 \mathrm{TeV}$, accepted for publication in Phys. Lett. B, e-print 1011.1931 [hep-ex]; CDF Collaboration, Search for Higgs Bosons Produced in Association with b Quarks, CDF Public Note 10105, 2010.

[32] CDF and D0 Collaborations and Tevatron New Phenomena and Higgs Working Group, Combined CDF and DO upper limits on MSSM Higgs boson production in tau-tau final states with up to 2.2 $\mathrm{fb}^{-1}$ of data, CDF Public Note 10099, D0 Note 6036-CONF, 2010.

[33] D0 Collaboration, Search for charged Higgs bosons in top quark decays, Phys. Lett. B682 (2009) 278.

[34] CDF Collaboration, Search for charged Higgs bosons in decays of top quarks in p $\bar{p}$ collisions at $\sqrt{s}=1.96$ TeV, Phys. Rev. Lett. 103 (2009) 101803. 